Recepción: 20 / 04 / 2017

Aceptación: 20 / 05 / 2017

Publicación: 15 / 07 / 2017

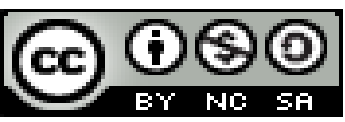

Ciencias de la Educación

Artículo Científico

\title{
Las actividades extracurriculares y el mejoramiento cualitativo del proceso de enseñanza-aprendizaje del idioma inglés
}

\section{Extracurricular activities and the qualitative improvement of the teaching-learning process of the English language}

Atividades extracurriculares e melhoria qualitativa do Inglês de ensinoaprendizagem 


\section{Resumen}

Este artículo se desarrolló mediante la aplicación de una modalidad de investigación de campo exploratoria, dado que analiza las variables que inciden en el proceso de enseñanza aprendizaje del idioma inglés. También es de tipo descriptivo y correlacional al establecer el grado de relación entre las variables estudiadas al comparar el comportamiento entre dos grupos de estudiantes en un ambiente controlado para cada escuela, por lo que encuadra en una investigación cuasiexperimental dado que no existe aleatoriedad en los sujetos objetos de estudio. Los resultados muestran la limitante que tienen las aulas numerosas y las estrategias didácticas pasivas, que no contribuyen con la motivación del estudiante en los colegios: Fiscales y Particulares José María Velasco Ibarra, 17 de Septiembre, San Francisco de Milagro y John Dewey, todos pertenecientes a la Ciudad del Milagro, Ecuador. Del análisis de los datos se ha llegado a una propuesta para la creación y aplicación de una guía de estrategias direccionada a mejorar el aprendizaje del idioma, con la intención de sentar un precedente que sirva de aporte a todas las comunidades educativas y a los profesores. En este contexto, las actividades extracurriculares son el andamiaje perfecto para vincular la motivación y el aprendizaje, particularmente por un idioma diferente al nativo. El proceso de enseñanza aprendizaje de la lengua ya sea extranjera o materna debe estar integrado con el currículo, ser consistente con las demandas lingüísticas, cognitivas, afectivas y culturales del contexto social del estudiante.

Palabras Claves: Enseñanza; didáctica; inglés. 


\begin{abstract}
This article was developed through the application of an exploratory field research modality, since it analyzes the variables that affect the teaching process of the English language. It is also descriptive and correlational when establishing the degree of relationship between the variables studied by comparing the behavior between two groups of students in a controlled environment for each school, which is part of a quasi-experimental research since there is no randomness in the subjects Objects of study. The results show the limitation of numerous classrooms and passive didactic strategies, which do not contribute to student motivation in schools: Prosecutors and Individuals José María Velasco Ibarra, September 17, San Francisco de Milagro and John Dewey, all belonging To the City of the Miracle, Ecuador. From the analysis of the data, a proposal has been reached for the creation and application of a strategy guide aimed at improving the language learning, with the intention of setting a precedent that serves as a contribution to all educational communities and teachers. In this context, extracurricular activities are the perfect scaffolding to link motivation and learning, particularly for a language other than the native language. The process of teaching - learning the language whether foreign or maternal must be integrated with the curriculum, be consistent with the linguistic, cognitive, affective and cultural demands of the student 's social context.
\end{abstract}

Key Words: Teaching; didactic; english. 


\section{Resumo}

Este artigo foi desenvolvido através da aplicação de uma forma de campo de pesquisa exploratória, uma vez que analisa as variáveis que afetam o processo de aprendizagem de Inglês. Também é descritivo e de correlação para estabelecer o grau de relacionamento entre as variáveis estudadas comparando-se o comportamento entre dois grupos de estudantes num ambiente controlado para cada tipo de ambiente escolar, de modo a caber numa pesquisa quase experimental como não há nenhuma aleatoriedade em indivíduos objectos de estudo. Os resultados mostram o fator limitante com inúmeras salas de aula e estratégias de ensino passivos que não contribuem para a motivação dos alunos nas escolas: Tax and Special José María Velasco Ibarra, 17 de setembro de San Francisco de Milagro e John Dewey, todos pertencentes a cidade de Milagro, Equador. A análise dos dados atingiu uma proposta para a criação e implementação de um guia de estratégia dirigido para melhorar a aprendizagem de línguas, com a intenção de criar um precedente que servirá como uma contribuição para todas as comunidades educacionais e professores. Neste contexto, as atividades extracurriculares são o andaime perfeito para vincular a motivação e aprendizagem, particularmente por uma língua nativa diferente. O processo de ensino - aprendizagem da língua estrangeiros ou mãe deve ser integrado com o currículo, ser coerente com as exigências linguísticas, cognitivas, emocionais e contexto social cultural do aluno.

Palavras-chave: Educação; ensino; inglês. 


\section{Introducción}

El considerar el mundo de la educación como una de las bases fundamentales de la sociedad lleva a analizar la importancia de fomentar el trabajo de actividades dentro del aula a través de metodologías activas y extracurriculares que motiven a los estudiantes a aprender en especial otro idioma, es así el caso del inglés. Educar no es simplemente transferir conocimientos, sino formar a los niños como hombres y mujeres del mañana, para que puedan y sepan vivir en sociedad. Debemos educar en todos los aspectos, la educación ha de ser integral, es decir, humana, ética, moral, física. Sin embargo, nos encontramos con una sociedad caracterizada por la falta de tiempo, aspecto que influye considerablemente en el ámbito escolar, y una elevada cantidad de materias/asignaturas que se imparten para llegar a obtener unos objetivos mayoritariamente conceptuales que realmente no aportan de manera significativa en este sentido.

Es una realidad la pobre aplicación de estrategias extracurriculares en la enseñanza de las lenguas extranjeras, esto deriva en un escaso conocimiento y manejo del de inglés en los colegios fiscales y particulares del cantón del Milagro, Ecuador. De allí surge la inquietud de realizar el presente estudio sobre el aspecto educativo, es decir, las formas más idóneas e inteligentes de construir una alternativa válida aceptada por los estudiantes de octavo año.

Este trabajo describe tales estrategias metodológicas y cómo se pueden aplicar por los docentes en las labores de enseñanza de las asignaturas de lenguaje extranjero en los procesos áulicos. En función de ello se consideró como ámbito de investigación a los estudiantes de octavo año de educación general básica de los Colegios José María Velasco Ibarra, Colegio 17 de Septiembre, Colegio San Francisco de Milagro y Colegio John Dewey del Cantón Milagro. 
En este orden de ideas, se logró una contextualización de la situación sociolinguíistica en el cantón Milagro, haciendo una breve referencia histórica de las teorías de educación y su fundamentación, y por último las variables planteadas y la hipótesis que sustentan esta propuesta didáctica.

Este análisis abordó la problemática, para conocer y analizar las causas de bajo rendimiento escolar y el abandono del sistema educativo. En la actualidad esto constituye una necesidad que debe ser resuelta, si se quiere incidir en el mejoramiento de la calidad y garantizar a futuro la equidad social, pues de no hacerlo conllevaría a otros problemas como son: La pobreza y la exclusión social que afecta a la población más joven del país.

Aunque no es difícil convenir en que la escuela no es el único lugar donde se gesta y provoca este problema, quizás tengamos que reconocer, sin embargo, que ella representa el orden institucional que crea las condiciones suficientes para que exista, ya que le toca construirlo y sancionarlo a tiempo.

Por consiguiente este artículo está dirigido a disminuir factores de riesgo del fracaso escolar mediante la aplicación de propuestas de intervención, en donde se prevé desarrollar acciones y procesos de carácter remedial o compensatorias para atención de estudiantes, así como, de ser necesario, la implementación de correctivos en el ámbito académico, esperando con ello contribuir a la disminución en el corto plazo y a la erradicación por completo en el futuro de las causas que inciden en el desinterés estudiantil por el dominio del idioma inglés.

\section{Materiales y métodos.}

Las actividades extracurriculares son programas que satisfacen dos condiciones básicas:

1) No son parte del programa curricular regular de la escuela. 
2) Tienen cierta estructura (no sólo para socializar sino para tratar de realizar una misión o meta social).

Las actividades extracurriculares son el andamiaje perfecto para vincular la motivación y el aprendizaje, por ello se propone el establecer un sistema, con el objetivo de despertar la atención, estimular la relevancia de los temas y generar confianza en los estudiantes hacia los logros proyectados mediante el estímulo de la satisfacción que brinda el aprendizaje de cosas nuevas.

Es lógico, entonces, que el aprendizaje de los estudiantes sea la clave para la selección y uso de un extenso abanico de estrategias de enseñanza. Las actividades de enseñanza/aprendizaje son los medios por las cuales los estudiantes se comprometen a aprender en esferas tanto cognitivas, afectivas, como de conducta o comportamiento (Cooper, 1999).

Las actividades extracurriculares tienen los siguientes objetivos:

- Posibilitar la ampliación del horario de los colegios.

- Complementar la formación de los alumnos con actividades de acuerdo al currículum y proyecto educativo del centro.

- $\quad$ Fomentar el desarrollo de valores y actitudes.

- Descargar tensiones de los alumnos.

En consecuencia, estos hallazgos se alcanzan mediante una investigación de campo por estar enmarcada en cuatro escuelas, y es de tipo descriptiva y correlacional en aras de poder medir el grado de asociación entre las variables, mediante herramientas estadísticas de correlación. De allí se pasaría a explicar el comportamiento de las variables, usando una metodología cuantitativa, estudiando el porqué de las causantes de la ausencia de actividades extracurriculares en la escuela. Desde esta 
perspectiva se pudo avanzar hacia un modelo exploratorio cuasiexperimental en vista de que se manejaron dos variables precisas: actividades extracurriculares y el mejoramiento cualitativo del proceso de enseñanza - aprendizaje.

En este orden de ideas, se usaron instrumentos tipo encuesta cerrada para la recolección de datos que permitiesen llegar a las conclusiones presentadas en este artículo.

Por tratarse de cuatro colegios se aplicó un muestreo estratificado que arrojó un ámbito de estudio de 172 estudiantes con un nivel de confianza del $95 \%$.

\section{Resultados}

Un $43 \%$ los estudiantes expresó que casi siempre les agrada la asignatura de Inglés, existiendo un $28 \%$ que manifestó agradarles algunas veces. Es importante observar la incertidumbre general que genera la materia. No obstante, cuando se plantea el aprendizaje por nuevas vías, un 59\% los estudiantes les gusta trabajar con grabadora, proyector de videos, dinámicas y juegos acordes a la asignatura, considerando que esto les ayuda a facilitar el aprendizaje del Inglés. Cobra aquí fuerza la necesidad de las actividades extracurriculares. Por otra parte, el $45 \%$ de los estudiantes encuestados en las instituciones educativas del Cantón Milagro manifestaron que nunca les hace escuchar su maestra(o) de inglés canciones u otras cosas que faciliten el aprendizaje. Aquí se muestra en parte la escasa cantidad de herramientas que el docente usa en el cumplimiento de los objetivos del curso. Más revelador todavía es el caso de la lectura del libro asignado como herramienta principal de aprendizaje del idioma, sólo un $43 \%$ dice leerlo revelando su corto alcance como motivador del aprendizaje en el estudiantado. 
En lo concerniente a la capacidad del aula para albergar a estudiantes, el $30 \%$ de los estudiantes indica que no es bueno el exceso de estudiantes en las aulas porque consideran que el maestro no puede monitorear todo el grupo en una forma idónea.

Para el caso de los docente casi la mitad está de acuerdo en que el aula no reúne las condiciones para un aprendizaje adecuado del idioma inglés, y es casi unánime, un $71 \%$ para ser precisos el que acepta la necesidad de la actividad extracurricular y un asombroso $100 \%$ de los maestros que avalan el diseño y empleo de un manual en este sentido.

\section{Recomendaciones}

- Aplicar un abanico de Estrategias Didácticas Extracurriculares que les ayude tanto al estudiante como al docente a facilitar el aprendizaje del idioma Inglés.

- Implementar los laboratorios de inglés con todos sus accesorios.

- Aplicación las estrategias de la guía propuesta como: Video juegos, Karaoke, rompecabezas, Juegos, Contar historias, tareas individuales y grupales etc.

- Racionalizar el número de estudiantes hasta un máximo de 25 por considerarlo un promedio apropiado donde el profesor puede monitorearlos en su totalidad.

- Realizar nuevas investigaciones aplicando otras estrategias extracurriculares

\section{Conclusiones}

Haciendo un balance de lo anterior, podemos concluir que: 
Si bien el uso de las estrategias extracurriculares no está presente en la normativa ni en la práctica educativa, es necesario incluirlo, porque en la actualidad se lo está haciendo de modo débil e incluso disperso, por lo que se requiere fortalecer una serie de aspectos normativos, institucionales y técnicos relacionados con la enseñanza integral de este tema.

Luego de revisado cada uno de los objetivos planteados, analizado el problema, la hipótesis se concluye. Que existe un 5\% de la población estudiantil de los centros educativos del Cantón Milagro que no les agrada el idioma Inglés por considerarlo complicado, esto se pudo apreciar al realizar las encuestas a instituciones fiscales como particulares, además se pudo evidenciar que existe un $28 \%$ de los estudiantes que algunas veces les agrada este idioma, lo que demuestra que hay una apatía por aprender una nueva lengua a pesar de que el Inglés es considerado de mucha importancia en la educación de nuestro país.

Otro factor que se considera de influencia en el aprendizaje del Inglés se debe a que los estudiantes en un $43 \%$ no leen otros libros sobre esta asignatura y solo se basan en libro que da el Ministerio de Educación, existiendo poco interés por investigar otras fuentes de consulta.

Al considerar las herramientas como estrategias para el aprendizaje un 59\% de los estudiantes manifestaron que les agrada trabajar con grabadoras, juegos, proyectores y videos, pero un $25 \%$ no les gusta trabajar con este tipo de materiales por considerarlos de poca ayuda en su aprendizaje.

El $12 \%$ de los docentes solo aplican algunas veces estrategias con actividades apropiadas al momento de explicar una clase haciendo que se dificulte el aprendizaje en los estudiantes provocando que estos muestren apatía por la asignatura. 
Otro factor que influye en el aprendizaje son las aulas numerosas que existen en especial en los colegios Fiscales donde hay promedios que alcanzan de sesenta y setenta estudiantes por aula lo que dificulta un monitoreo total del curso.

La poca y casi nula implementación de laboratorios de Inglés en estos centros de estudio hace que no se pueda aplicar listening como estrategias útiles para el aprendizaje del idioma Inglés.

Otra causa es la no utilización de otros libros de inglés interactivos que les ayude a ampliar sus conocimientos.

Según los datos obtenidos se afirma que el poco interés de los estudiantes por aprender un segundo idioma y la falta de aplicación de estrategias extracurriculares es una realidad palpable en los centros educativos investigados, específicamente en los Colegios Fiscales José María Velasco Ibarra, Colegio 17 de Septiembre, Colegio Particular San Francisco de Milagro. En el Colegio particular John Dewey, existe un mayor porcentaje de estudiantes que prestan interés a la asignatura por ser una institución bilingüe, la problemática que se presenta en la mayoría de los colegios se puede minimizar con la aplicación de una Guía de estrategias, con actividades apropiadas para ayudar a los estudiantes a interesarse por el idioma Inglés y a mejorar el aprendizaje por ende obtener un mejor rendimiento académico.

Los estudios efectuados, la elaboración y las respuestas en las encuestas, además de los extraídos de las fuentes bibliográficas se pueden hacer una verificación de la hipótesis, donde se puede aclarar que desde el punto de vista no se estaba muy lejos de la realidad existente.

\section{Variable independiente}

"Con la adecuada aplicación de actividades extracurriculares." 
La investigación demostró que existía un alto porcentaje de estudiantes que no les agrada el idioma Inglés a causa de la desmotivación por aprender otro idioma esto incide que se produzca la deserción escolar y hasta pérdida del año lectivo.

\section{Variable Dependiente.}

“Mejorará el aprendizaje del idioma Inglés por parte de los estudiantes del 8vo año de Educación Básica del Cantón Milagro”.

En análisis de las preguntas planteadas, la información teórica, estadística presentada en los capítulos anteriores, se pudo conocer que la gran importancia que tiene para los estudiantes y docentes la aplicación de una Guía de estrategias extracurriculares lo cual ayudará a mejorar el aprendizaje por ende el rendimiento escolar de los estudiantes en las instituciones educativas involucradas en la investigación.

\section{Bibliografía}

Andrade. (2009). Aprendizaje del Inglès. En Andrade, Aprendizaje del Inglès. Esmeraldas: Homer.

Argudo. (2006). Realidad en el mundo de la Educacion. En Argudo, La Educaciòn. Guayaquil: Holguìn.

Austin. (2009). Actividades de enseñanza. En Austin, Deja que el mundo entre en el Aula. Morata: Publicaciones Venture.

Banchòn. (11 de Diciembre de 2006). http://xserra.net/unica/2006/oce-2005/Documents/Treballs/ind/46.pdf, "Importancia de la investigación". Extraìdo el 9 de Mayo del 2011, del sitio web: http://xserra.net/unica/2006/oce.

Barros. (21 de Noviembre de 2007). Extraído el 2 de Julio de 2011, del Sitio Web: http://portal.educar.org/foros/estrategias-de-ensenanza.

Brand. (21 de Junio de 2008). Extraido el 16 de Abril de 2011, del sitio web:www.monografias.com/trabajos19/estrategias/aprendizaje/estrategias-aprendizaje.shtml.

Cabrera. (7 de Enero de 2009). Extraido el 18 de Mayo de 2011, del Sitio Web: http://www.buenastareas.com/ensayos/Estrategias-Didacticas-Para-La-Ense\%C3\%B1anza-Del/853868.html.

Caiza. (2008). Actividades extracurriulares. Mèxico: Publicaciones Lens. 
Cervantes. (s/f). Karaoke: un instrumento globalizador para la enseñanza de lenguas. Extraído el 16 de diciembre del 2011.- disponible en: cvc.cervantes.es/enseñanza/biblioteca_ele/asele/pdf/.../04_0523.pdf

Cooper, J. (1999). Estrategias de enseñanza. Guía para una mejor enseñanza. México: Limusa Noriega Editors.

Ford, L. (1985). Pedagogía ilustrada. Principios generales. El Paso, TX: Editorial Mundo Hispano.

Garcìa. (15 de Diciembre de 2006). Extraido el 4 de Junio de 2011, del sitio web http://www.elmundo.es/yodona/estaticas/especiales/2008/09/colegio/extras.html.

Hynds, S. (1994). Making connections. Language and learning in the classroom. Norwood, MA:

Christopher-Gordon Publishers, Inc.

La técnica del Rompecabezas- Portada Principal. Extraído el 15 de diciembre del 2011. Del sitio web: www.rmm.cl/index_sub.php?id_seccion=2270\&id_portal...

Laboratorio de IdiomasenSecundaria2 Dic. 2007. Ejercicios y actividades de aprendizaje de vocabulario (Inglés ) Extraído del sitio web:

jlcabello.wordpress.com/

Leontiev, A. A. (1981). Psychology and the learning process. Oxford: Pergamon Press.

Muestreo de una población. Extraído el 22 de Noviembre del 2011, del sitio web:www.ingenieria.peruv.com/...muestreo/muestreo_estratificado.htm.

Montiel. (18 de Julio de 2009)bibliotecavirtual.clacso.org.ar/ar/libros/becas/1999/vazquez.rtfRecuperado el 15 de Junio de 2011, de bibliotecavirtual.clacso.org.ar/ar/libros/becas/1999/vazquez.rtf.

McDonough, S. H. (1981). Psychology in foreign language teaching. London: George Allen \& Unwin.

Merriam, S. B. (1988). Case study research in education. A qualitative approach. San Francisco, CA: JosseyBass Publishers.

Noriega. (15 de Febrero de 2005).

http://www.colegiohumboldtcaracas.com/site/index.php?sec=21. Extraido el 3 de Mayo de 2011, del sitio web: http://www.colegiohumboldtcaracas.com/site/index.php?sec=21.

Peralta. (5 de Octubre de 2009). Importancia del idioma Inglès. Extraìdo el 26 de Abril de 2011, del sitio web: http://www.misrespuestas.com/porque-es-importante-el-idioma-ingles.html.

Peralta. (2009). Ventajas de las actividades extracurriculares. Mèlida: Publicaciones Holguìn.

Pérez, J, M. A. C., \& T. Chacón, D. C. (2011). El podcast como innovación en la enseñanza del inglés como lengua extranjera. Pixel-Bit, Revista de Medios y Educación, (39),

Robles. (7 de Junio de 2009). http://aureadiazgonzales.galeon.com/. Recuperado el 9 de Abril de 2011. Disponible en:http://aureadiazgonzales.galeon.com/

Richards, J. C., y Rodgers, T. S, (1992). Approaches and methods in language teaching. A description and analysis. Cambridge: Cambridge

University Press.

Smith, F. (1981). Demonstrations, engagement, and sensitivity: The choice between people and programs. Language Arts, 58, 643-642. 
Stiefel. (2008). Nuevo Paradigma educativo. En Stiefel, Nuevo Paradigma educativo (pág. 21).

Vite. (8 de Abril de 2009). Extraido el 25 de Mayo de 2011, del sitio web: http://www.definicionabc.com/general/cualitativo.php.

Wells, G. (1986). The meaning makers. Children learning language and using language to learn.

Portsmouth, NH: Heinemann.Muest estraficado(s.f) 